\title{
More evidence of substructure in the motions of nearby halo stars
}

\author{
P. Re Fiorentin ${ }^{1}$, A. Helmi ${ }^{2}$, M. G. Lattanzi ${ }^{3}$ and A. Spagna ${ }^{3}$ \\ ${ }^{1}$ Dipartimento di Fisica Generale, Università di Torino, Via Pietro Giuria 1, 10125 Torino, I, \\ email: paola.re.fiorentin@to.infn.it \\ ${ }^{2}$ Kapteyn Instituut, P.O. BOX 800, 9700 AV Groningen, NL, \\ ${ }^{3}$ INAF, Osservatorio Astronomico di Torino, Via Osservatorio 20, 10025 Pino Torinese, I
}

\begin{abstract}
We explore the stellar halo of the Milky Way to search for fossil signatures of past mergers. We use the Beers et al. (2000) catalog of non-kinematically selected metal poor stars in the solar neighborhood to select subsets of halo stars within 1-2 kpc of the Sun. Motivated by the results of high resolution CDM simulations, we look for substructure in the kinematics of the fastest stars. When a two-point velocity correlation function is applied to these subsets, statistical evidence of substructure is found. This appears to be due to a small moving group with dynamical properties similar to the stellar "stream" previously discovered by Helmi et al. (1999).
\end{abstract}

\section{Introduction}

Observations of metal-poor stars have often been used to discriminate between alternative galaxy formation and evolution scenarios (e.g. Eggen, Lynden-Bell \& Sandage 1962; Searle \& Zinn 1978). In a CDM Universe, the first objects to form are small systems which then merge and give rise to the larger scale structures we observe today. This theory predicts the presence of substructures due to the mergers and accretion that galaxies may have experienced over their lifetime.

Several examples of mergers and galaxy interactions have been observed in the Milky Way, such as the disrupted Sagittarius and Canis Major dwarf galaxies (e.g. Ibata, Gilmore \& Irwin 1995; Martin et al. 2004), the phase-space stream of halo stars in the solar neighborhood (Helmi et al. 1999), the tidal tails from halo globular clusters and dwarf spheroidal galaxies (Odenkirchen et al. 2001).

\section{Evidence of substructures in the Solar neighborhood}

The analysis of a high resolution simulation of the formation of a dark matter halo by Helmi et al. (2002) has shown that dark matter particles are smoothly distributed in space in the equivalent of Solar neighborhood. While their kinematics can be represented by a smooth multivariate Gaussian distribution, the motions of the most energetic particles are strongly clumped and anisotropic.

Motivated by this result, we turned to the extensive catalog of metal-poor stars, selected without any kinematic biases, compiled by Beers et al. (2000). This sample provides proper motions, radial velocities, and distance estimates for 1258 stars with a wide range of metal abundances. We identified a set of halo stars defined as those having metal abundances $[\mathrm{Fe} / \mathrm{H}] \leqslant-1.5 \mathrm{dex}$, while we excluded those with typical disk kinematics (coplanar and circular orbits, $\mathrm{L} \sim \mathrm{L}_{\mathrm{z}}, \mathrm{V}_{\phi} \sim 220 \mathrm{~km} \mathrm{~s}^{-1},|\mathrm{z}| \leqslant 1.5 \mathrm{kpc}$ ). Our "clean" halo sample contains 186 and 392 stars within 1 and $2 \mathrm{kpc}$ of the Sun, respectively, and includes subdwarfs, giants, and variable stars (Re Fiorentin et al., in preparation). 

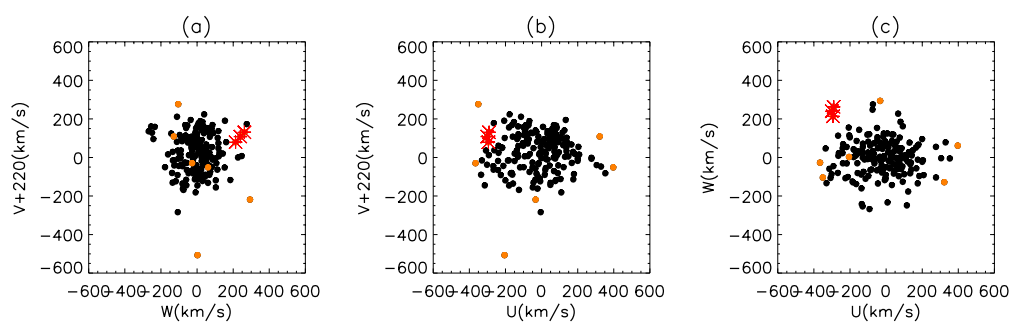

(d)

Figure 1. Distribution of the selected sample of halo stars within $1 \mathrm{kpc}$ of the Sun. (a-c) Velocity projections in the $(\mathrm{U}, \mathrm{V}, \mathrm{W})$ space; (d) Velocity direction: the position of each particle is given by the spherical angular coordinates $\boldsymbol{\alpha}=(\phi, \theta)$ of the velocity vector. Of the 186 stars present in this volume, the $5 \%$ fastest are highlighted in gray. Among them, the asterisks identify the objects with velocity difference less than $100 \mathrm{~km} \mathrm{~s}^{-1}$.
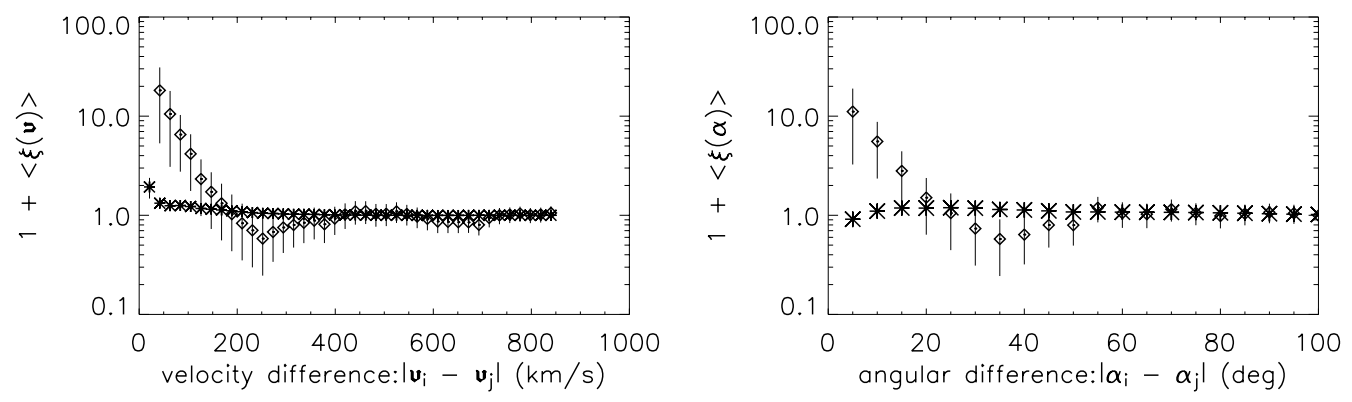

Figure 2. The two-point velocity correlation function $\xi(v)$ (left panel) and angular correlation function in velocity space $\xi(\alpha)$ (right panel) for the $1 \mathrm{kpc}$ sample of halo stars (asterisks) and for the subset of $5 \%$ fastest stars (diamonds). Error bars are based on Poissonian counts.

The velocity distribution of the subset of halo stars within $1 \mathrm{kpc}$ is relatively smooth, however this seems no longer to be the case when we focus on the fastest objects, as shown in Fig. 1. The kinematics of the $5 \%$ fastest moving stars (gray dots) seem more clumped and anisotropic.

We quantify the deviations from a smooth Gaussian distribution due to kinematic substructures by computing the two-point velocity correlation function, defined as

$$
\xi=\frac{\langle D D\rangle}{\langle R R\rangle}-1
$$

where $\langle D D\rangle$ is the number of pairs of stars in our data with velocity difference in a given bin, and $\langle R R\rangle$ is defined analogously for the same number of random points drawn from a multivariate Gaussian distribution convolved with expected observational errors, and averaged over 100 realizations. $\xi(v)$ measures the excess of pairs of stars moving with given velocity difference, above that expected from a random sample. $\xi(\alpha)$ measures the excess of pairs of stars moving in the same given direction range, above that expected from a random sample.

Clumping due to kinematic substructures (i.e. groups of stars moving with similar velocities and directions) appears as an excess at small velocity difference (see Fig. 2). This signal, clearly much stronger for the subset of $5 \%$ fastest objects, is due to a moving group formed by 3 stars denoted by the asterisks in Fig. 1.

The distribution in the space $\mathrm{E}, \mathrm{L}_{\mathrm{z}}$ and $\mathrm{L}_{\mathrm{xy}}$ of the selected set within $2 \mathrm{kpc}$ of the Sun shows that our main kinematic clump and the stars of the stream identified by Helmi 

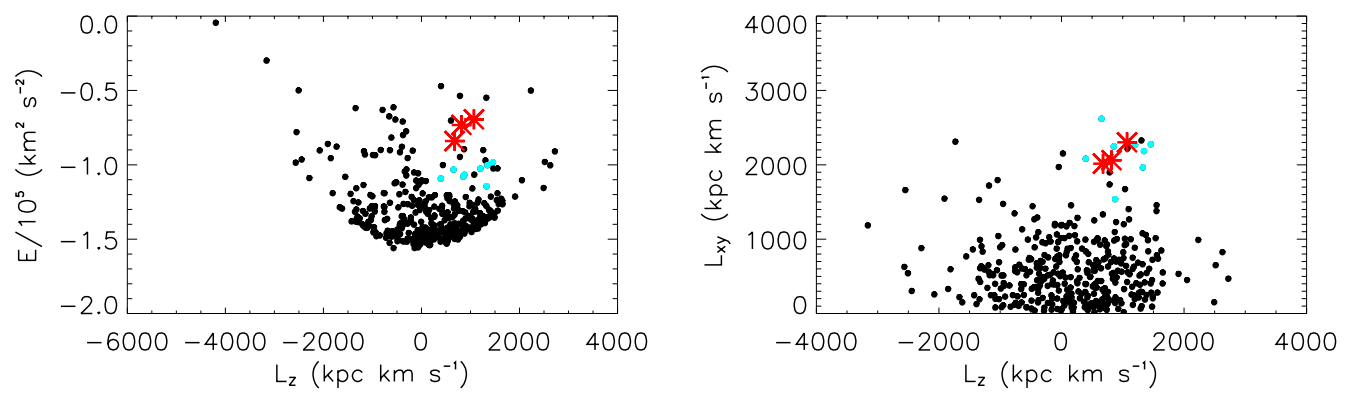

Figure 3. Distribution of the selected sample of 392 halo stars within $2 \mathrm{kpc}$ of the Sun in the space of adiabatic invariants. The asterisks denote the stars of our main kinematic clump, the gray dots denote the stars of the stream identified by Helmi et al. (1999).

et al. (1999) have similar momenta but somewhat different energies (see Fig. 3). This could imply that these groups have different origins. However, it seems also plausible that these groups have been stripped off from the same progenitor in different passages.

\section{Conclusions}

We have found evidence of substructure in the motions of the fastest stars in the Solar neighborhood, at a level which seems to be consistent with that predicted by high resolution simulations of dark matter halos in a hierarchical universe.

The moving group identified may represent a subsample of a stellar stream spread out along the orbit of a disintegrated body, but the number of stars available is still too small for any definite conclusion.

In the inner halo, where most of the merger activity is expected to have taken place, currently available samples with accurate kinematics are still quite small. Space astrometric missions, such as GAIA, will collect samples of millions of stars with very precise positions and velocities which will dramatically improve the situation.

\section{Acknowledgements}

We wish to thank Volker Springel and Simon White for permission to use their simulations; Antonaldo Diaferio for many useful comments. P.R.F. thanks the Sterrekundig Instituut Utrecht for hospitality during her visit. Partial financial support came from the Italian Ministry of Research (MIUR) through the COFIN-2001 program.

\section{References}

Beers, T. C., Chiba, M., Yoshii, Y., Platais, I., Hanson, R. B., Fuchs, B. \& Rossi, S. 2000 Astrophys. J. 119, 2866-2881.

Eggen, O. J., Lynden-Bell, D. A. \& Sandage, A. R. 1962 Astrophys. J. 136, 748-766.

Ibata, R. A., Gilmore, G. \& Irwin, M. J. 1995 Mon. Not. R. astr. Soc. 277, 781-800.

Helmi, A., White, S. D. M. \& Springel, V. 2002 Phys. Rev. D 66, 063502.

Helmi, A., White, S. D. M., de Zeeuw, P. T. \& Zhao, H. S. 1999 Natur. 402, 53-55.

Martin, N. F., Ibata, R. A., Bellazzini, M., Irwin, M. J., Lewis, G. F. \& Dehnen, W. 2004 Mon. Not. R. astr. Soc. 348, 12-23.

Odenkirchen, M., et al. 2001 Astrophys. J. 548, L165-L169.

Searle, L. \& Zinn, R. 1978 Astrophys. J. 225, 357-379. 